\title{
Certain Investigations on Reversible Image Watermarking
}

\author{
T. Sujatha*, Susan Augustine and J. Granty Regina Elwin \\ Department of Information Technology, Sri Krishna College of Engineering and Technology, \\ Coimbatore -641008,Tamil Nadu, India; sujatha@skcet.ac.in, \\ susanaugustine@skcet.ac.in, grantyreginaelwinj@skcet.ac.in
}

\begin{abstract}
Objectives: To protect secret information in a digital medium without compromising robustness. There are many approaches introduced regarding the importance of utilizing medical, military and natural images for image watermarking procedure. Reversible watermarking is appreciated that the original media can be recovered without any loss of information after extracting stego-data. With an aim to provide higher security and robustness, certain investigations have been done on reversible image watermarking. Methods/Statistics: This research work mainly addresses on privacy, reversibility, payload and robustness related goals. Considering these challenges, three parts of image watermarking techniques, namely proposed Bi-orthogonal Wavelet Transform and Importance Measure Model (BWT and IMM), introduced Dynamic Prediction Error Integrated Reversible Image Watermarking (DPEIRIW) and proposed Multi Wavelet Transform based and Oppositional Particle Swarm Optimization (MWT - OPSO) are introduced to improvise the data hiding performance. Findings: The methodologies introduced in this research work not only surpass other approaches with considerations to a variety of incursions, but still maintains a satisfactory image quality. From the experimental results, it is clearly understood that this suggested approach MWT and OPSO attains the average PSNR of $59.84 \mathrm{~dB}$ and average NC of 1, which is minimal when comparedwith the all the other existing strategies. Applications/Improvements: As the ongoing trend is now towards multi-core computing and the requirements for the real time video communication is very much in demand, the ownership right information also required to be preserved. It could be a great future direction to deploy this design approach under multi-core platforms.
\end{abstract}

Keywords: Correlation Coefficient, Entropy, Importance Measure Model, OPSO Biorthogonal DWT, Prediction Error, Reversible Watermarking, NC, PSNR

\section{Introduction}

The entrenchment of programedintelligence in whole aspects of day-to-day chores is one of the colossalhightechoccurrences of the past twenty years. Digital data can be savedmethodically with a very hugeendowment and it could be engineered very effortlesslyand effectuallyby using computers. Furthermore, withsuch formidabledata could be channelled in a quick and economical way through data transmission systems. Programmed intelligence struck much unique dominance over counterpart intelligence $\frac{1-3}{}$. Editing is simple because various locations can be accessed that are in requirement of a difference.
Plagiarizing is also easy with no deprivation of integrity and is exact to the aboriginal. With deploy intermediadispensation over the World Wide Web, substantiation is highly intimated than ever due to the liability of boundless replication. High risk for information creators is established due to the simple way of transference and stratagem of digital data initiations. So the consortium holders need is to be remunerated each and every instance their work is being used. Additionally, they see to that their work is not altered in the absence of their knowledge. For digital data, consortium constraints and silence the substantiation are exceedingly tough tasks ${ }^{4}$.

*Author for correspondence 
One solution is using some encryption techniques to avoid the unauthorized access. Any how, ascription doesn't issue complete precaution. Once the decryption is done for the engraftedintelligence, they could be openly being accessed or modified. The above give constrain can be resolved bycloaking some proprietorship intelligence into the intermedia intelligence, which could be catheterized later to manifest the proprietorship. The watermarking model has been stipulated so far as an attainable elucidation when, in a definite appeal circumstance, there is the need to implant an informative data in a digital documentation in an inconspicuous way ${ }^{5,6}$. Usually, when proceeding with receptive images such as deep planetary science, counterinsurgency research and medical analysis, the ultimate- consumer can't put up with wager to get gnarled data. One illustration above all: a radiological physicist who is examining a radioactivity illustration to authorize if assertive pomology is prompt or not. It can't be customary that his interpretation is faulty both, primarily, to care for the victims health and secondarily, to safeguard the efforts of the radiological physicist himself. In that case, irretrievable watermarking algorithms doesn't seem to be viable. Due to this stern prerequisite, additional departments of watermarking technique has been imported which is catalogued as vagrant, where, with this terminology, it would be anticipated that the important content, except the watermark signal, is retrieved back from the watermarked output file such that any test can be conducted on the primal data. Hence, by working out like this, the strategy is termed as a zero-impact but allows conveying necessary message. The Reversible watermarking method is also termed as invertible or lossless and was considered to be practiced mainly in conditions where the genuineness of a digital image has to be approved and the original content is peremptorily used at the decoding side $e^{\frac{7-11}{}}$.

Channelizing secure processed data was the most challenging task before the technological invention of steganography and cryptography. Hackers favour to distort the primal outcome either by altering it or by using the same methodology to make benefit without giving award to the owner. Thus, privacy proficiency needs to be well competent, potent and uncommon to decrease malicious. Hence, a very significant watermarking technology emerged.

Deploy watermarking is a relatively new field of the last two decades. Deploy information can be embedded in data and extracted later. The watermarking information can be texts, logos, handwritten signatures or numbers, and have many applications. It is noticed that one of the base necessities for deploy watermarking is to preserve the standard of the initial facts not being gnarled when a watermark is fixed into it. Additionally, there are several other factors that are needed for particular application. One basic requirement of deploy watermarking is the necessity to continue the original condition of distorted content. Perceptual transparency, robustness ${ }^{12}$, capacity to restore data without inceptive, binary unit rate of data indexing algorithms, secured watermarking, confirmation of proprietorship are a few requirements tend to vary with respect to watermarking applications.

One of the watermark challenges is to maintaining excellent invisibility peculiarities. Another fundamental requirement is stalwartness; the watermark should be defying to illegitimate malformation and decryption. Additionally, the watermark should be liberal to picture transforming methods like confining (lossless and lossy) and calculated experiments to extirpate the watermark. Another challenge of watermark algorithm is imperceptibility. A capable watermark arrangement is the one that can counter balance preservation, and durability. Another watermark challenge is to implement a watermark system that can handle immense content amplitude while accommodating the condition of the watermarked pictures. Watermarked images may be subject to a number of common attacks.

In order to meet the above said challenges, it is required to develop and propose novel watermarking algorithms for embedding the images and suitable recovery. Thus, this work focused on developing watermarking algorithms to meet the required challenges. The ultimate goal of this research work is to improve current digital watermarking technique and design it for specific applications. It is not possible to craft a watermarking procedure that strives in all scenarios and can confront all the aggressions. Here it is aimed to design effective and cost efficient digital watermarking systems for different applications and different requirements.

Currently, several governments have been encouraging e-Health memorandum, which gather and preserve distinctive health facts in a computerized form. These records contain sensitive personal information and should be protected. For pharmaceutical pictures, distinctive databases are basically preserved in an independent file which can be looked through at the very instant time 
with the pictures. Nonetheless, this information is usually not preserved and could be penetrated by strangers.

A recent incident involving accessing patient information for job screening in the University of Health Network in Toronto raised the alarm on the issue of privacy protection and access control ${ }^{\frac{13}{3}}$. Digital watermarking could be used to embed important messages into the covert case. The expediency of watermarking is that the content are kept within the primal cases, which reduces the chance of it being terminated or changed manipulated. In addition,as watermarking acquaints only tiny misinterpretation, it wouldn't cause adversities for physicians who are not provided with the permission to look into the distinctive cases. One impediment of digital watermarking is that the primal case is completely altered after the watermark is introduced. For pharmaceutical pictures, these alterations may not be alluring no matter how unnoticeable it is.

The fundamental goal of this research work is to proffer lossless watermarking or reversible watermarking scheme to address these problems. A reversible watermarking is a method in which both the original copy and the watermark image could be recovered without any losses. Bi-orthogonal Discrete Wavelet Transform based techniques are used in the proposed schemes due to its superior performance over other transforms and the segment blueprint which is shown in Figure 1.

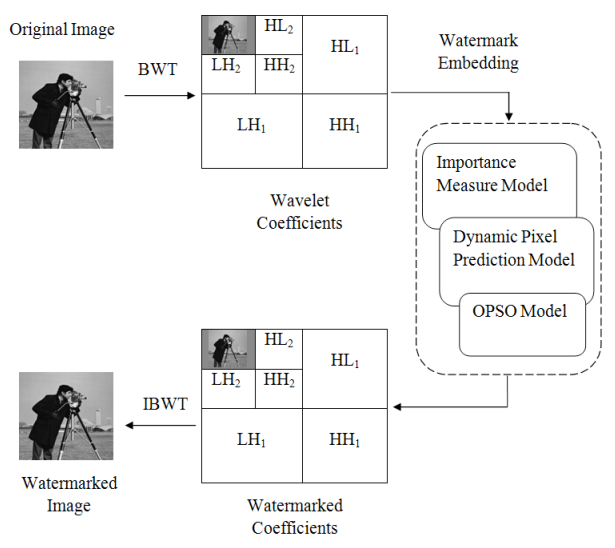

Figure 1. Block diagram of the scheme with Bi-orthogonal Discrete Wavelet Transform technique.

\section{Research Background}

In the field of digital watermarking, the image watermarking predominantly had engrossed a huge amount of sympathy in the analysis part of association. The majorpart of the analysis work is devoted to image watermarking in comparison with aural and music videos. Some of the reasons are described.

- The test images are readily available.

- Images carry sufficient redundant information so that watermarks can be embedded easily.

- It may be fictitious that any fortunate image watermarking breakthrough may be innovated for the videos also.

Pictures are interpreted in dimensional realms and also in frequency realms. The image part in the altered domain is specified in terms of its frequency coefficients and in spatial domain; it is defined by picture elements. Simply, alter realm means the image in the form of multiple frequency bands. To represent an image in the transform domain, capricious transfigures like Discrete Cosine Transform (DCT), Discrete Wavelet Transform (DWT) or Discrete Fourier Transform (DFT) can be used. Every of these transfigures has its endemic features and represents the picture in its own manner. Watermarks could be imposed within pictures by changing the alter dominion frequency coefficient bands. In case of the structural dominion, plain watermarks can be imposed in the pictures by changing the picture element rate or the Least Significant Bit (LSB) values. Nonetheless, more potent watermarks can be imposed in the alter domains of pictures by changing frequency coefficients. $\operatorname{In}^{14}$ proposed a research paper titled "Secure Spread Spectrum Watermarking for Multimedia" and after that majority part of the analysis work is done based on this work. After that, transform domain watermarking blueprints has emerged and now it is Reversible Water marking $\frac{15,16}{}$.

An analysis of article proposes that the problem rests in the area of digital watermarking in conniving blueprints suitable for both grey scale and colour images that are more vigorous towards a huge horizons of intrusion and sustain feasible picture standards especially in medical and military data. Most of the blueprints out perform especially when image misinterpretation or confining ratio is huge. Since in the multimedia digital communication, tamper detection has more serious commercial implications than the copyright control, it has been decided to go with robust watermarking. Out of all the multimedia contents such as image content, audio content and video content, any image watermarking algorithm which is 
lucrative can be extended as video watermarking, hence, image watermarking is intended. With future augmentation in thought, the overlay intermediate chosen is a picture. In the first part of this investigation, a reversible image watermarking algorithm by applying Dual orthogonal Detached Wavelet Transform was proposed. Initially, the picture was resized and disintegrated into frequency sub bands. The best band and ideal locations in the best band those are apt for enclosing is construct alongside with the service of degenerating measure. Consequently, the expected watermark inserting procedure is accomplished to insert a watermark in the cover picture.

In the succeeding parts of this investigation, a capricious picture watermarking blue print established on Dynamic Forecast Flaw and Opposition Particle Swarm optimization methodology is proposed. Wherein, watermark embedding is done by the calculation of Prediction Error and Fitness value. In addition, Multi Wavelet Transform is applied for choosing of optimal location for embedding. The performance efficiency metric of proposed image watermarking schemes is examined in terms of Crest Alarm to Sound Proportion, and Standardized interrelationships values. From the empirical outcomes, it is clearly inferred that this proposed scheme has obtained better results when compared to other approaches available in the literatures $\frac{17-19}{}$.

\section{BWT and IMM}

In this proposed work, an irresolute picture watermarking procedure applying Dual-Orthogonal Wavelet Transfigure and Priority Method Figure is planned. The two important factors in the watermarking procedure is watermark enclosing and watermark eradication. In the enclosing procedure, primarily Dual-orthogonal Distinct Wavelet Transfigure is indulged to decay the initial given picture into variety rings and the superiorring is selected compared with the degeneration. Consequently, the selecting of a correct area is done by using Priority Method Figure. After selecting the superiorring and the area for enclosing, the suggested enclosing procedure is doing new here Single-bit picture is used as a watermark.

\subsection{Election of the Ring}

The process for electing the ring is given. Primarily, the authentic picture is brought back to the intended preapproved quality size. Let the authentic picture after re-sizing is proposed by of size $M \times N$. After re-sizing, 2D DWT implying the Dual-orthogonal Transfigure is done to have a Coefficient Approximate (CA) and Accurate Coordinated Rings (Coefficient Horizontal $\mathrm{CH}$, Coefficient Vertical - CV, Coefficient Detail - CD). Here, CA rings coordinates will be ignored as they can't be exampled for the watermark enclosing procedure. From the comprehensive ring, sequent the optimal ring is chosen using the degeneration rate. The degeneration rates are chosen for the $\mathrm{CH}, \mathrm{CV}$ and $\mathrm{CD}$ rings and are characterized by $E_{c h}, E_{c v}$ and $E_{c d}$ respectively. Based on the Degeneration, the superior band $X$ is chosen for upcoming procedures.

That is, the/ring with the highest degeneration rates is chosen as the optimal ring and is chosen for upcoming procedures and portrayed as in Equation 3.1.

$$
E_{X}=\operatorname{Maximum}\left(E_{c h}, E_{c v}, E_{c d}\right)
$$

Here, $E_{X}$ is the degeneration of the chosenring $\mathrm{X}$. After the given picture is re-sized to a global size, then Dual-orthogonal DWT is used and the optimal ring for enclosing with the help of degenerative measure ${ }^{20}$.

\subsection{Collection of Areas}

When the superior ring is chosen, the next thing to do is finding the optimal location where the embedding can take place. To achieve this, the Importance Measure Model. The exemplary takes into application five criterion of concentration, Comparison, Area, agitation and balance. For each criterion, corresponding attention patterns are determined and ultimately the doubled sum is shown as the global Important Value.

The picture is primarily segregated into parts and the Important Values is determined for each and every of the parts. And then those parts with an acceptable global Important Values are selected as the Area for enclosing. Let the picture given for searching the area is shown as $H$ of dimension $m \times n$. Let the count of parts be $y$ and each part $\left(B_{i} ; 0<i<y\right)$ is with dimension $p \times q$. That is. $m \times n$ $=y \times p \times q$ Subsequently, the Important Measure $\left(D_{i} ; 0<\right.$ $i<y)$ corresponding to each block is computed. The individual Importance Values of specifications is calculated compared on the amount of interest that a normal human can have for that particular region .

In the extraction process, initially converse distinct wavelet transfigure is applied and then the eradication process is done in the area chosen. While investigating the 
performance of BWT and IMM, this suggested method achieved a set of acceptable outcomes having a moderate PSNR amount of 39.8606 and highest NC amount of 1 . The robust feature of the suggested watermarking scheme is calculated with the help of a variety of extracting methods and acceptable outcomes were reached in every aspect. The processing time period elapsed for the run of the proposed scheme algorithm with respect to the considered image data sets is 83 seconds.

\section{DPEIRIW Model}

Dynamic Prediction Error Integrated Reversible Image Watermarking (DPEIRIW) is developed and implemented for the considered image samples from the database. The proposed approach consists of four calibres, namely preprocessing module, pixel prediction module, enclosing module and extraction module. The part diagram of the modelled approach is as given in Figure 2.

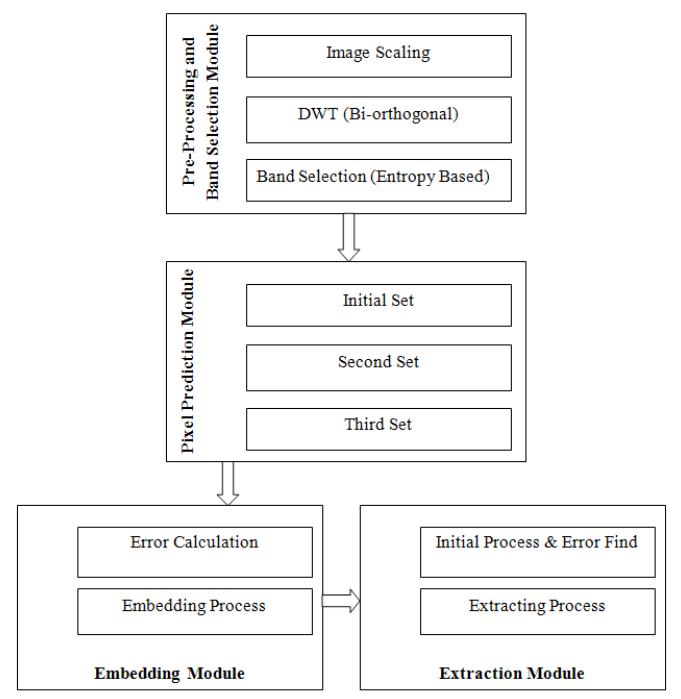

Figure 2. DPEIRIW scheme.

\subsection{Pixel Prediction Module}

In this module, the pixel values are predicted based on the position and making use of mathematical operations. The prediction is carried out based on the adjacent pixel values and the median is calculated out. The process of this prediction depends on the position of the pixel and it is taxonomies accordingly. The classes include four kinds of pixels based on the position termed as initial set, first derived set, second derived set and third derived set. Based on the prediction fault, embedding and extraction can be carried out. Once the image is predicted, the prediction fault is found out by correlating the predicted image to that of the original value and embedding is carried out based on the error value. Owing to a high correlation between the adjacent pixels, error would be small and pixels having lower errors are preferred for embedding process. For extraction, the Prediction error is the digression between the predicted picture element amount and the watermarked picture element value. This error value is compared with a set threshold to extract the watermark.

The evaluation metrics wormed are Peak Signal to Noise Ratio (PSNR) and Normalized Correspondence (NC). The high NC and PSNR obtained for the technique with an average NC of 0.9948 and PSNR of 49.6594. This suggested procedure has performed well by having higher PSNR and NC values in comparison to other approaches. The algorithm processing time elapsed for the run of the proposed method algorithm with respect to the considered image data sets is 81 seconds.

\section{MWT - OPSO Model}

The primary focus of this proposed research work is to design an efficient, powerful reversible image watermarking scheme depends on MWT-OPSO Model. The explanation is illustrated in Figure 3. The scheme composed of mainly two modules such as embedding and filtering. In the embedding step, the original source image is splitter into different bands based on Haar Wavelet Transform, in which low frequency band sub division is selected and then the Dual-orthogonal Wavelet Transfigure is used to decay the picture. Out of all rings, the approximation band is left unapproachable and degeneration of various rings are enumerated. The highest band is chosen as the ring and from that, the superior location is sorted out using OPSO algorithm. The performance efficiency of the proposed research work is analyzed with many grey-level images inclusive of medical images. The investigation cadets used are Peak Signal to Noise Ratio and Normalized Correlation. From the investigation results, it is found that the maximum PSNR of 59.86, NC of crisp 1 and average embedding capacity of 1024 bits is gained in the proposed watermarking scheme which shows clearly that the quality of the image is not compromised. The running time elapsed for the processing of the proposed algorithm with respect to the considered image data sets is 71 seconds. 


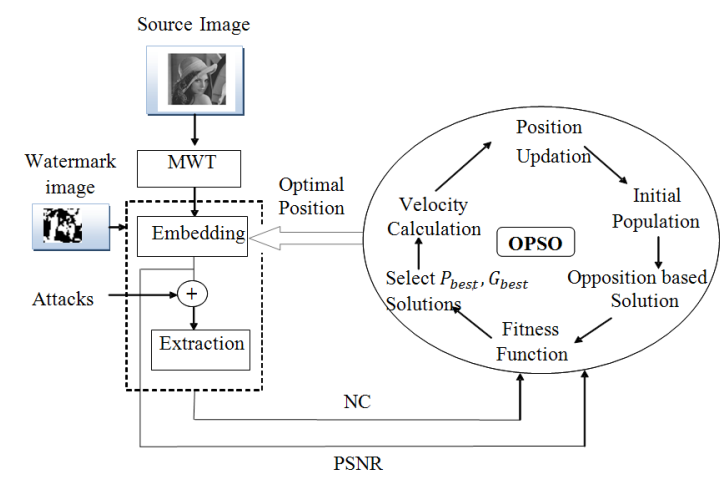

Figure 3. Hybrid MWT and OPSO Watermarking System Model.

\subsection{Optimal Position Selection}

The Particle Swarm Optimization (PSO) algorithm is a facile developmental procedure which is very different from the other developmental procedures where it initiates the incitement of public characteristics. PSO has presented high standard of effectiveness in searching fine answers to developmental problems. PSO is a populace-dependent examining procedure and begins with a starting populace of aimlessly achieved outcomes called fragments. Each fragments in PSO has a location and a momentum. PSO keeps in mind both the optimal location constructed by all the fragments and the optimal location constructed by every fragments in the finding process. One challenge that we come across in the standard PSO is that it could without any difficulty slip into provincialapogee in a lot of developmental challenges. To have an upper hand on this challenge, in this analysis the Opposition based PSO (OPSO) methods shows how to neglect the incomplete concurrence and allows OPSO to go on with the inquest for Standardized apogee by implying opposition-based learning. The basic concept of Opposition Based Learning (OBL) is the deliberation of a conclusion and its analogous opposite conclusion continuously to estimate the current applicants' outcome. The suggested accession for populace initializing used by the resistance placed procedure in which the populace and its adverse populace are taken as input. The correctness of both populaces is judged and the superior one is chosen. Finally, the watermark image is imposed onto the source picture to obtain the watermarked picture ${ }^{21}$.

\section{Performance Comparison}

The basic idea behind this investigation work is changeable pictures watermarking similar on the proposed Multi
Wavelet Transfigure based and Oppositional Particle Swarm Optimization method algorithm (MWT and OPSO). The main motivation behind this approach is to employ the capability of MWT in yielding high embedding capacity of algorithm, enhanced data hiding security measure and improved quality of watermarked image content. Here, the performance efficiency of the proposed approach is proved by comparing the developed hybrid MWT and OPSO technique with that of the Weighted Median Approach ${ }^{22,23}$ Coordinate Prediction Approach ${ }^{24}$, Optimal Channel selection approach $\underline{25}, \underline{26}$ Shear let domain approach ${ }^{27}$ and that of the earlier methods BWT and IMM and DPEIRIW proposed.

\section{Conclusion}

Reversible watermarking requires high embedding capacity, content distortion which should be as low as possible and robustness against attacks. These conflicting requirements carve the path for this research work. Considering these challenges, a reversible watermarking scheme resistant to geometrical attacks was proposed in this investigation. Here, the maximum PSNR of $59.8466 \mathrm{~dB}$ is computed for the brain Magnetic Resonance Imaging (MRI) image. From the results it is clearly understandable that this proposed approach is having better attainment than that of existing approaches available in the literature. The proposed watermarking scheme does not require the original image for extracting the watermark. This investigation aimed at creating a scheme, robust to geometrical distortions, compression and filtering attacks. This research work mainly addresses privacy, reversibility, payload and robustness related goals. To achieve these goals BWT and IMM, DPEIRIW and MWT and OPSO methods were proposed.

\section{Future Scope}

Based on this investigation, a few areas of further research work are identified as follows:

a. Further improvement could be possible, wherein the same approaches could be practiced on the video files of different types. Applying this technique on MPEG, H.264 and 3D video files could be a future guidance for this research.

b. As the ongoing trend is now towards multi-core computing and the requirements for the real time video 
communication is very much in demand, the ownership right information also required to be preserved. It could be a great future direction to deploy this design approach under multi-core platforms.

c. In addition to that, the research can be protracted from strict authentication to soft authentication. Along with authentication verification, small secret data such as session keys can also be imposed in encrypted format and traded between two communication ends. Hence, authentication and confidentiality can also be achieved together.

\section{References}

1. Vanco BM, Christensen JL. Ego depletion increases regulatory success in educational digital media environments. Computers in Human Behavior. 2017; 62:602212. https:// doi.org/10.1016/j.chb.2016.04.031

2. Sohrabi B, Iraj H. Implementing flipped classroom using digital media: A comparison of two demographically different groups' perceptions. Computers in Human Behavior. 2017; 60:514-24. https://doi.org/10.1016/j.chb.2016.02.056

3. Orzech KM, Grandner MA, Roane BM, Carskadon MA. Digital media use in the $2 \mathrm{~h}$ before bedtime is associated with sleeps variables in university students. Computers in Human Behavior. 2016; 55:43-50. https://doi.org/10.1016/j. chb.2015.08.049

4. Qi X, Xin X. A singular-value-based semi-fragile watermarking scheme for image content authentication with tamper localization. Journal of Visual Communication and Image Representation. 2015; 30:312-27. https://doi.org/10.1016/j. jvcir.2015.05.006

5. Cox I, Miller M, Bloom J, Fridrich J, Kalker T. Digital watermarking and steganography. Morgan Kaufmann; 2007. 624. https://doi.org/10.1016/B978-012372585-1.50015-2

6. Shih FY. Digital Watermarking and steganography: Fundamentals and techniques. CRC Press; 2007.

7. $\mathrm{Hu} \mathrm{Y,} \mathrm{Jeon} \mathrm{B.} \mathrm{Reversible} \mathrm{visible} \mathrm{watermarking} \mathrm{and} \mathrm{lossless}$ recovery of original images'. IEEE Transactions on Circuits and Systems for Video Technology. 2006; 16(11):1423-9. https://doi.org/10.1109/TCSVT.2006.884011

8. Khan A, Siddiqa A, Munib S, Malik SA. A recent survey of reversible watermarking techniques. Information Sciences. 2014; 279:251-72. https://doi.org/10.1016/j.ins.2014.03.118

9. Feng $\mathrm{G}$, Qian Z, Dai N. Reversible watermarking via extreme learning machine prediction. Neuro Computing. 2012; 82:62-8. https://doi.org/10.1016/j.neucom.2011.10.028
10. Chen CC, Tsai YH. Adaptive reversible image watermarking scheme. Journal of Systems and Software. 2011; 84(3):428-34. https://doi.org/10.1016/j.jss.2010.11.891

11. Chen M, Chen Z, Zeng X, Xiong Z. Model order selection in reversible image watermarking. IEEE Journal of Selected Topics in Signals Processing. 2010; 4(3):592-604. https:// doi.org/10.1109/JSTSP.2010.2049222

12. Cox IJ, Miller ML, Bloom JA, Honsinger C. Digital watermarking. Springer; 2002. https://doi.org/10.1016/ B978-155860714-9/50009-2

13. Chen TPC, Chen T. Progressive image watermarking. IEEE International Conference on Multimedia and Expo. 2000; 2:1025-8.

14. Cox IJ, Miller ML. Review of watermarking and the importance of perceptual modeling. Electronic Imaging. 1997; 97:92-9. https://doi.org/10.1117/12.274502

15. Hu HT, Hsu LY. A mixed modulation scheme for blind image watermarking. AEU-International Journal of Electronics and Communications. 2016; 70(2):172-8. https://doi.org/10.1016/j.aeue.2015.11.003

16. Hu HT, Chang JR, Hsu LY. Robust blind image watermarking by modulating the mean of partly sign-altered DCT coefficients guided by human visual perception. AEUInternational Journal of Electronics and Communications. 2016; 70(10):1374-81. https://doi.org/10.1016/j. aeue.2016.07.011

17. Sujatha T, Geetha K. Reversible image watermarking using bi-orthogonal wavelet transform and importance measure model. Journal of Theoretical and Applied Information Technology. 2014; 60(3):531-42.

18. Sujatha T, Geetha K. Dynamic prediction error integrated reversible image watermarking. Asian Journal of Information Technology. 2016; 15(17):3311-19.

19. Sujatha T, Geetha K. Multi wavelet transforms and oppositional particle swarm optimization for an efficient reversible image watermarking. International Journal of Advanced Engineering Technology. 2016; 7(2):978-87.

20. Hu HT, Hsu LY, Chou HH. Variable-dimensional vector modulation for perceptual-based DWT blind audio watermarking with adjustable payload capacity’. Digital Signal Processing: A Review Journal. 2014; 31:115-23. https://doi. org/10.1016/j.dsp.2014.04.014

21. Hu X, Shi Y, Eberhart RC. Recent advances in particle swarm. IEEE Congress on Evolutionary Computation. 2004; 1:90-7.

22. Naskar R, Chakraborty RS. Performance of reversible digital image watermarking under error-prone data communication: A simulation-based study. IET Image Processing. 2012; 6(6):728-37. https://doi.org/10.1049/ietipr.2011.0160 
23. Naskar R, Chakraborty RS. Reversible watermarking utilizing weighted median-based prediction. IET Image Processing. 2012; 6(5):507-20. https://doi.org/10.1049/ietipr.2011.0244

24. Naskar R, Chakraborty RS. Reversible image watermarking through coordinate logic operation based prediction. International Conference on Information Systems Security; 2011. p. 190-203. https://doi.org/10.1007/978-3-64225560-1_13

25. Huynh-The T, Banos O, Lee S, Yoon Y, Le-Tien T. Improving digital image watermarking by means of optimal channel selection. Expert Systems with Applications. 2016; 62:17789. https://doi.org/10.1016/j.eswa.2016.06.015

26. Khan A, Malik SA, Ali A, Chamlawi R, Hussain M, Mahmood MT, Usman I. Intelligent reversible watermarking and authentication: Hiding depth map information for 3D cameras. Information Sciences. 2012; 216:155-75. https://doi.org/10.1016/j.ins.2012.06.014

27. Wang CP, Wang XY, Xia ZQ, Zhang C, Chen XJ. Geometrically resilient color image zero-watermarking algorithm based on quaternion Exponent moments. Journal of Visual Communication and Image Representation. 2016; 41:247-59. https://doi.org/10.1016/j.jvcir.2016.10.004 